\title{
Red blood cell distribution width in patients with limb, chest and head trauma
}

Giuseppe Lippi ${ }^{1}$, Chiara Bovo², Ruggero Buonocore ${ }^{3}$, Michele Mitaritonno ${ }^{4}$, Gianfranco Cervellin ${ }^{4}$

\author{
${ }^{1}$ Section of Clinical Biochemistry, University of Verona, Verona, Italy \\ 2University Hospital of Verona, Verona, Italy \\ ${ }^{3}$ Laboratory of Clinical Chemistry and Hematology, Academic Hospital of Parma, \\ Parma, Italy \\ ${ }^{4}$ Emergency Department, Academic Hospital of Parma, Parma, Italy
}

Submitted: 8 February 2016

Accepted: 18 March 2016

Arch Med Sci 2017; 13, 3: 606-611

DOI: https://doi.org/10.5114/aoms.2017.67282

Copyright $\odot 2017$ Termedia \& Banach

\section{Abstract}

Introduction: This study investigated the values of red blood cell distribution width (RDW), an emerging and independent predictor of morbidity and mortality, in patients with limb, chest and head trauma.

Material and methods: The study sample consisted of all patients who attended the emergency department (ED) of the University Hospital of Parma for limb, chest and head traumas requiring admission to hospital wards during the year 2014. The controls consisted of outpatients living in the same geographical area and undergoing routine laboratory testing for health check-up.

Results: The final study sample consisted of 290 patients with limb $(n=97)$, chest $(n=49)$ or head $(n=144)$ trauma and 967 outpatients. Significantly increased RDW values at admission were observed in all trauma patients compared with controls. Although the frequency of increased RDW (>14.6\%) was higher in all trauma patients than in controls, a subanalysis revealed that increased RDW values were significantly more frequent in patients with head trauma than in controls, but not in those with limb or chest trauma. In multivariate analysis, a significant association was found between head trauma and hemoglobin $(p<0.001)$ or RDW $(p=0.005)$. Head trauma patients had a $\sim 3$-fold higher likelihood of increased RDW values than controls. The negative and positive predictive values of increased RDW for predicting the presence of head trauma were $0.90(95 \% \mathrm{Cl}: 0.88-0.92)$ and $0.24(95 \% \mathrm{Cl}$ : $0.19-0.30)$.

Conclusions: The results of this study highlight that RDW is increased in patients admitted to the ED with head trauma.

Key words: trauma, injury, anisocytosis, red blood cell distribution width.

\section{Introduction}

According to recent statistics of the US Centers for Disease Control and Prevention (CDC), as many as 41 million emergency department (ED) visits and 2.3 million hospital admissions for different types of trauma are recorded each across the nation [1]. Overall, trauma injuries account for approximately $30 \%$ of years of potential life lost in the US, a value that is 2-fold and 3-fold higher than the years of potential life lost due to cancer and heart disease, thus generating a yearly expenditure of nearly $\$ 585$ billion, including both health care costs and lost productivity. In-

\author{
Corresponding author: \\ Prof. Giuseppe Lippi MD \\ Section of Clinical \\ Biochemistry \\ University of Verona \\ Piazzale L.A. Scuro, 10 \\ 37121 Verona, Italy \\ Phone: 0039-045-8124308 \\ E-mail: giuseppe.lippi@univr.it
}


terestingly, trauma injuries are ranked first among the leading causes of death up to 46 years of age, and the third leading cause of death across all age groups. The leading causes of trauma include falls (32\%), motor vehicle traffic accidents (14\%), being struck by or against objects or persons (11\%) and unintentional injuries (8\%) [1]. As regards our country (i.e., Italy), the number of severely injured patients requiring hospitalization was found to be as high as 391 per million per year, with an overall mortality approximating $25 \%$ and increasing with the age of the patients [2]. Male patients aged 18-64 years were more subjected to occupational injuries, trauma on the road and violence than others, whereas women were more vulnerable to domestic injuries and self-inflicted violence [2].

Due to these worrying epidemiological data, the initial assessment, management and disposition of trauma patients in the ED is now emerging as a considerable component of the overall workload in this healthcare setting [3]. Several lines of evidence now attest that the most efficient management of trauma patients in the ED encompasses a timely and thoughtful analysis of the patient's situation, by means of clinical examination, diagnostic imaging and laboratory testing [4]. Indeed, the availability of rapid diagnostic tests that may allow safe triage and immediate risk stratification of the single patient should be regarded as a valuable perspective for more efficient utilization of resources in short stay units such as trauma centers and emergency rooms [5].

Anisocytosis is conventionally defined as the presence of red blood cells (RBC) with a large heterogeneity of volumes, and is usually reported as RBC distribution width (RDW) in terms of standard deviation (RDW-SD) or coefficient of variation (RDW-CV) of erythrocyte sizes, the latter being expressed as the percentage of RDW-SD divided by the mean corpuscular volume (MCV) [6]. Depending on the hematological analyzer, the upper limit of the reference range of RDW-CV varies between $14.0 \%$ and $15.0 \%$, with a reference value of $14.6 \%$ identified in many clinical trials, as reported elsewhere [7]. Recent evidence attests that the introduction of RDW in diagnostic algorithms may be useful in the initial evaluation of patients with a vast array of human disorders such as cardiovascular disease [8], venous thrombosis [9], cancer [10] and severe infections [11], but is also a powerful and independent predictor of clinical outcomes, including short- and long-term mortality [12].

Therefore, the aim of our study was to investigate whether RDW assessment at ED admission may be useful for the clinical reasoning and triage of patients with limb, chest and head traumas, which are the most frequent types of traumas observed in the ED.

\section{Material and methods}

In this retrospective investigation, the study sample consisted of all consecutive patients aged 18 years or older visited in the ED (a level II trauma center) of the University Hospital of Parma (Italy) during the entire year 2014 for limb, chest and head traumas, who needed to be admitted to hospital wards. The facility is a 1250-bed teaching university hospital, serving a population of approximately 450,000 inhabitants. The diagnoses of trauma were systematically extracted from local hospital records according to specific International Classification of Diseases-9 (ICD-9) codes and related diagnostic terms. The information was then reviewed by two expert physicians for deleting wrong or dubious records, which mainly occur for typing errors of ICD-9 codes. No patient had received prehospital blood transfusion before hematological testing at ED admission. The control sample consisted of outpatients aged 18 years or older and living in the same geographical area (i.e., Parma province), who underwent routine laboratory testing for health check-up during the same period (i.e., the entire year 2014). The complete blood cell count (CBC), hence entailing hemoglobin, MCV and RDW-CV, was performed in whole blood samples $(13 \times 75,2.0 \mathrm{ml}$ plastic whole blood tube containing spray-coated $\mathrm{K}_{2}$ EDTA; Becton Dickinson Italia S.p.A., Milan, Italy) collected from trauma patients before receiving any type of treatment and from outpatients during the health check up, respectively. The analysis was performed within $2 \mathrm{~h}$ from blood collection, using an identical hematological analyzer (Sysmex XE-2100, Sysmex Co., Kobe, Japan). The quality and comparability of results were guaranteed by routine performance of internal quality controls and participation in an external quality assessment scheme.

\section{Statistical analysis}

Since the Shapiro-Wilk test revealed that data were non-normally distributed, the results were finally reported as median and interquartile range $(\mathrm{IQR})$ or percentage. The significance of difference was analyzed with the Mann-Whitney test (for continuous variables) and Pearson's $\chi^{2}$ test with Yates' correction (for categorical values). A multivariate linear regression analysis was also used, to identify potential associations with trauma. Diagnostic significance was estimated by means of the receiver operating characteristic (ROC) curve. The odds ratio (OR) with 95\% confidence interval $(95 \% \mathrm{Cl})$ was also calculated. The analyses including RDW values (expressed as RDW-CV) were based on dichotomous exposures, using the instrument-specific cut-off value of $14.6 \%$ [13]. The level of statistical significance was set at 


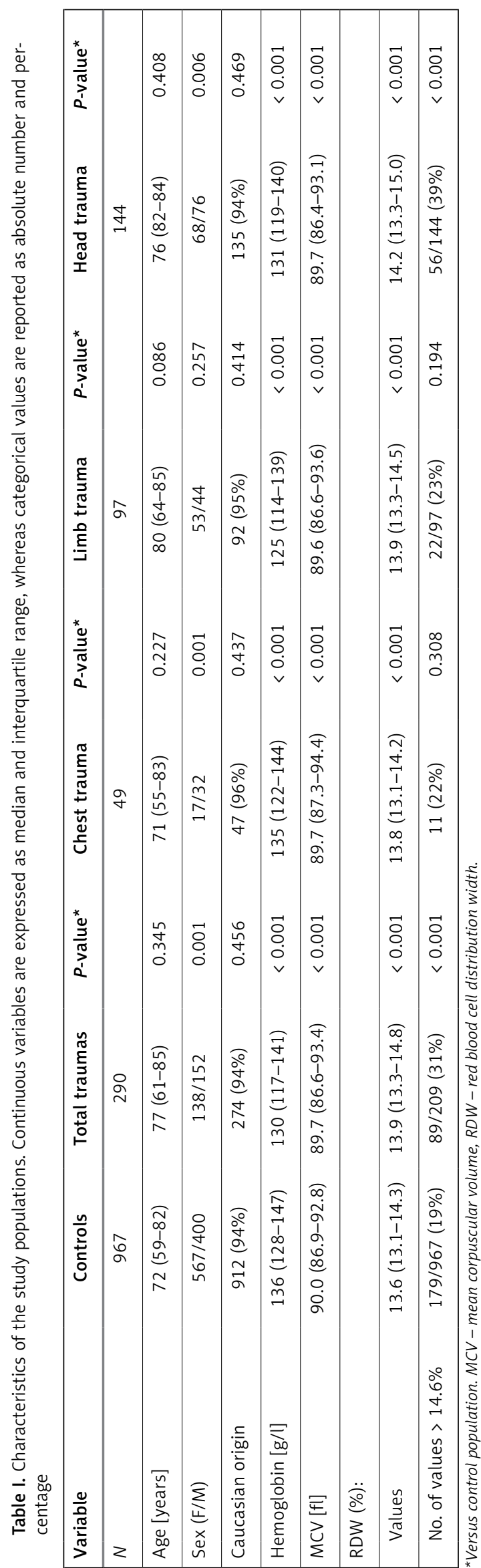

$p<0.05$. The statistical analysis was performed using Analyse-it (Analyse-it Software Ltd, Leeds, UK) and MedCalc Version 12.3.0 (MedCalc Software, Mariakerke, Belgium). This retrospective study was carried out in accordance with the Declaration of Helsinki, under the terms of relevant local legislation, and was cleared by the institutional review board of the Academic Hospital of Parma.

\section{Results}

After hospital database extraction, 316 records of patients visited in the ED with limb, chest and head traumas were identified. Twenty-six of these cases were excluded due to wrong or dubious registration. Therefore, 290 patients with limb $(n=97)$, chest $(n=49)$ and head $(n=144)$ traumas who required hospital admission were included in this study. The final sample of controls consisted of 967 outpatients.

The main characteristics of the study samples are shown in Table I. No statistically significant difference was observed for age and ethnic origin, whereas a greater number of women was present in the total sample of trauma patients, as well as in patients with chest and head trauma. Although the differences were modest overall, the values of hemoglobin, MCV and RDW were always higher in trauma patients than in controls. A significantly higher RDW value was also observed in patients with head trauma than in those with chest trauma, whereas no statistically significant difference was observed between limb trauma and both head and chest traumas (Figure 1). The effect size (ES) was small comparing controls with all cases (ES, 0.15), and with patients with limb (ES, 0.14) or chest trauma (ES, 0.10), whereas it was found to be medium comparing controls and patients with head trauma (ES, 0.25). The frequency of increased RDW values (i.e., > 14.6\%) was significantly higher in the entire sample of trauma patients than in controls. However, when the different categories of trauma patients were compared with controls, a higher frequency of increased RDW values was only observed in patients with head trauma but not in those with limb or chest trauma. Interestingly, the frequency of increased RDW was also significantly higher in patients with head trauma than in those with limb $(p=0.006)$ or chest trauma $(p=0.028)$. In multivariable linear regression analysis, in which the presence of a specific type of trauma was entered as the dependent variable and age, sex, hemoglobin, MCV and RDW were entered as independent variables, significant associations were found between head trauma and he$\operatorname{moglobin}(\beta=-0.004 ; p<0.001)$ or $\operatorname{RDW}(\beta=0.03$; $p=0.005)$, between limb trauma and hemoglobin $(\beta=-0.004 ; p<0.001)$, and between chest 


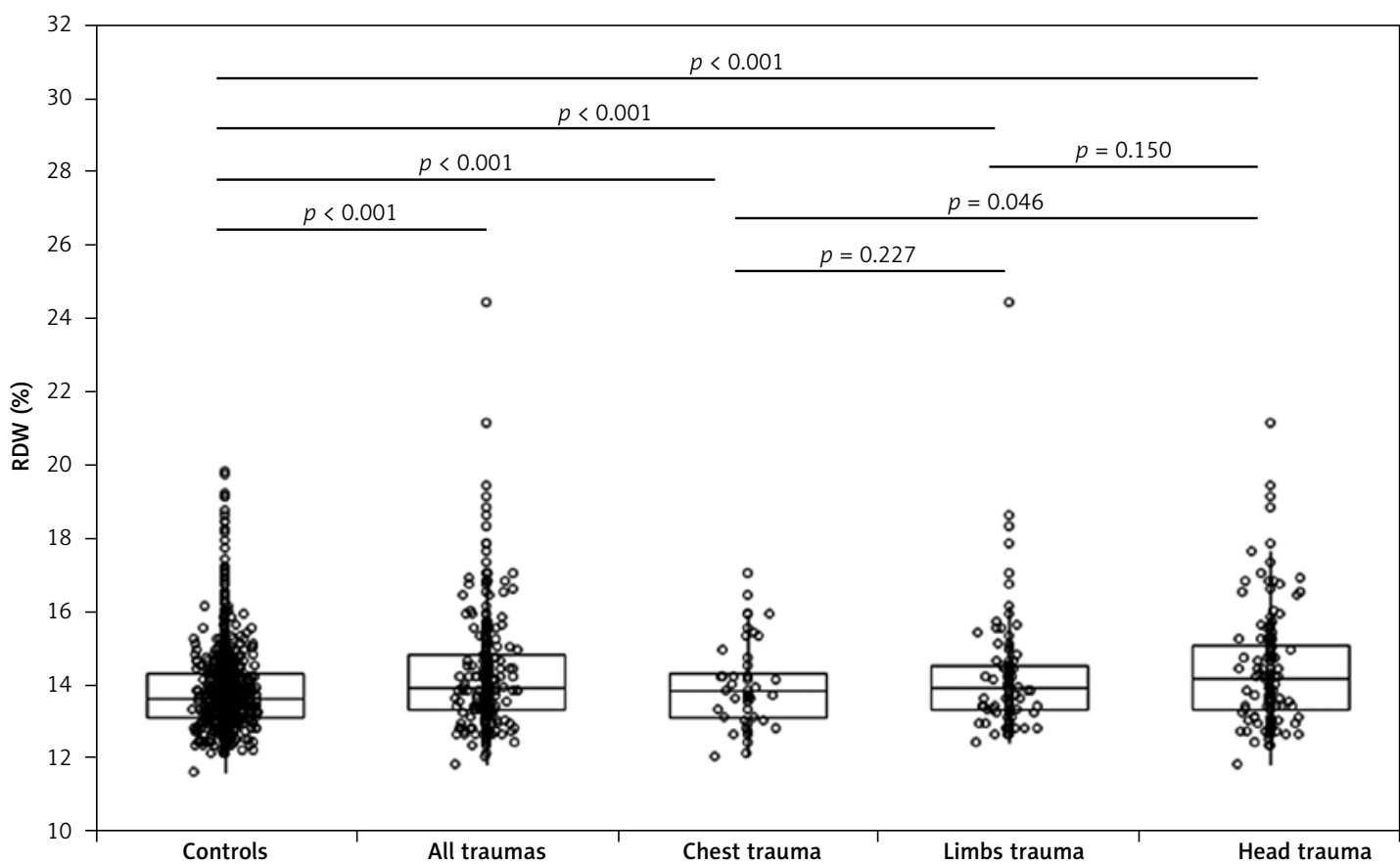

Figure 1. Values of red blood cell distribution width (RDW; median and interquartile range) in a control population of unselected outpatients and in patients with chest, limb and head trauma at emergency department admission

trauma and hemoglobin $(\beta=-0.002 ; p<0.001)$. Accordingly, patients with head trauma were found to have a nearly 3-fold higher likelihood of increased RDW values compared to controls $(\mathrm{OR}=2.80 ; 95 \% \mathrm{Cl}: 1.93-4.07 ; p<0.001)$, whereas the likelihood of increased RDW values was not statistically significant in patients with either chest $(\mathrm{OR}=1.27 ; 95 \% \mathrm{Cl}: 0.64-2.54 ; p=0.491)$ or $\operatorname{limb}(\mathrm{OR}=1.29 ; 95 \% \mathrm{Cl}: 0.78-2.13 ; p=0.318)$ trauma. In multivariable linear regression analysis, the RDW value was slightly but significantly correlated with the severity of the injury in patients with head trauma as established by positive findings (i.e., acute subdural, epidural or parenchymal hematoma, traumatic subarachnoid hemorrhage, cerebral contusion and brain swelling) in the computed tomography scan $(\beta=0.02 ; p=0.047)$.

Interestingly, the RDW value at admission exhibited an area under the curve (AUC) of 0.61 (95\% Cl: $0.56-0.66 ; p<0.001)$ for head trauma, 0.57 (95\% Cl: 0.51-0.63; $p=0.016$ ) for limb trauma and of 0.55 (95\% Cl: $0.46-0.64 ; p=0.130)$ for chest trauma, respectively. The negative and positive predictive values of increased RDW (i.e., $>14.6 \%)$ for predicting the presence of head trauma were $0.90(95 \% \mathrm{Cl}: 0.88-0.92)$ and 0.24 (95\% Cl: 0.19-0.30), respectively (Figure 2).

\section{Discussion}

Taken together, the results of our study attest that RDW values are higher in patients with chest, limb and head trauma compared to controls of the same geographical area, with the highest
RDW values being observed in patients with head trauma. The frequency of higher anisocytosis (i.e., RDW > 14.6\%) was also significantly higher in patients with traumas than in controls, but this difference seemed almost entirely attributable to the subset of patients with head trauma. Interestingly, the frequency of increased RDW values was nearly double in patients with head trauma compared to those with chest or limb trauma. Additional important information emerging from this study is the significant diagnostic performance exhibited by the RDW in head trauma (Figure 2),

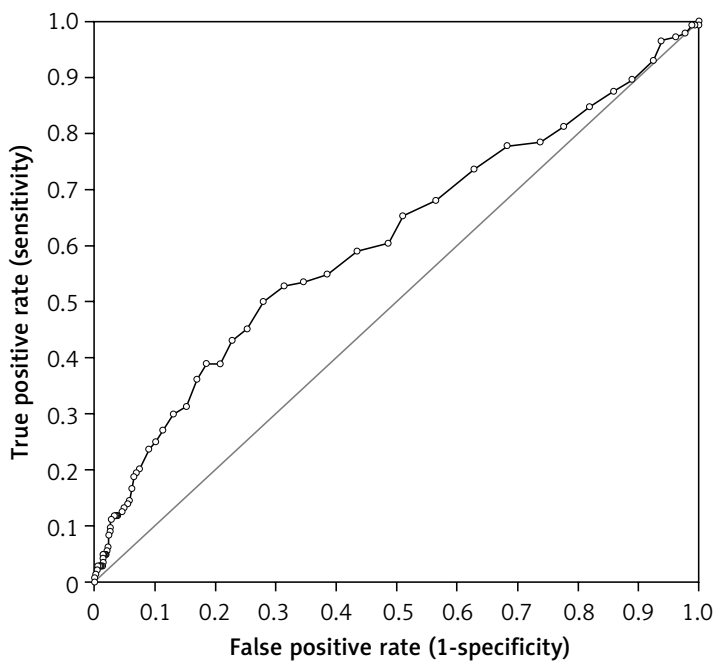

Figure 2. Receiver operating characteristic (ROC) curve of red blood cell distribution width (RDW) at emergency department admission in patients with head trauma 
along with the modest but significant association with the severity of trauma (i.e., the diagnosis of brain injury in CT). An AUC of 0.61 and, especially, a negative predictive value of 0.90 further support the potential significance of introducing RDW in diagnostic and prognostic algorithms in the ED. Indeed, RDW is a rather simple and inexpensive laboratory parameter not requiring special training or specific scoring systems to be interpreted, which can be rapidly obtained by means of traditional laboratory instrumentation, but also with point of care devices located in the emergency room.

The increasing overcrowding in EDs is posing serious threats to healthcare systems worldwide. In recent years patients wait times and periods of ambulance diversion have constantly increased, thus raising concern about the timeliness, appropriateness, safety and overall efficiency of triage and treatment [14]. Importantly, an increase of mortality, medication errors, pain, and length of stay, along with other negative effects, has been well documented as a consequence of overcrowding [15-17].

Some organizational and cost reduction strategies have been proposed for increasing the efficiency and appropriateness of care in emergency services [18], some of which entail the introduction and use of simple, rapid and inexpensive rule out tests, that would enable safe discharge of patients and reduce occupation [19, 20]. Several lines of evidence now attest that an intriguing relationship seemingly exists between anisocytosis and the occurrence of many human diseases and their prognosis [21]. However, the available scientific literature on anisocytosis in patients with traumas is scarce.

Majercik et al. studied 3,637 female and 5,901 male trauma patients with the RDW value available upon admission to a level I trauma center, who were followed up for 1 year [22]. An increased RDW value was found to independently predict 30-day and 1-year mortality in both sexes (hazard ratio (HR) between 1.17 and 1.43 per quintile in women, between 1.17 and 1.52 per quintile in men, respectively). Garbharran et al. studied 698 consecutive patients admitted to the hospital with hip fracture [23], and found that increased RDW values were significantly associated with in-hospital $(H R=1.12), 120$-day $(H R=1.13)$ and 1 -year mortality $(H R=1.13)$. In a subsequent study Zhang et al. retrospectively studied $122 \mathrm{pa}$ tients with traumatic brain injury [24], and found that RDW values were significantly higher in the non-survival than in the survival group $(12.9 \%$ vs. $12.2 \%$; $p$ 0.01). In another investigation, Paulus et al. measured RDW in shock trauma patients admitted to a level I trauma center [25], and reported that massive transfusion (i.e., administration of 10 or more packed red blood cells in the first $24 \mathrm{~h}$ ) was up to 3.5-fold more likely in patients in the highest quintile of RDW than in those in the lowest quintile.

As regards the putative mechanisms underlying the increased RDW values in trauma patients, it is still unclear as to whether RDW may be regarded as a direct cause or rather as an effect of many human diseases [6]. Indeed, anisocytosis has been frequently observed in patients with sustained oxidative stress and inflammation, two common features of trauma, especially of traumatic brain injury $[26,27]$. As such, the excessive generation of proinflammatory mediators and the imbalance between oxidant and antioxidant agents occurring after severe injuries may directly affect erythrocyte biology, thus ultimately impairing erythropoiesis or enhancing the heterogeneity of erythrocyte volumes in vivo [6].

In conclusion, the use of a clinical scoring system is crucial in the initial approach to patients with different types of traumas [28], in order to achieve a timely diagnosis and effective therapeutic management with both conventional and innovative treatments $[29,30]$. Therefore, the integration of simple and inexpensive biomarkers such as the RDW within validated diagnostic algorithms may be seen as a valuable perspective for initial patient evaluation. Although a definitive mechanistic relationship between anisocytosis and trauma remains to be defined (e.g., higher erythrocyte turnover or bone marrow stress during trauma), this is the first cross-sectional retrospective study showing that the RDW values are increased in trauma patients, especially in those with head trauma. Additional studies are needed to establish whether the use of this simple and inexpensive parameter combined with other factors as part of the risk assessment may be useful during initial evaluation at ED admission, in order to identify a subset of patients who may require more aggressive management in the trauma center.

\section{Conflict of interest}

The authors declare no conflict of interest.

\section{References}

1. Centers for Disease Control and Prevention. Trauma statistics. Available at: http://www.nationaltraumainstitute.org/home/trauma_statistics.html. Last access: 5 February 2016.

2. Chiara O, Mazzali C, Lelli S, Mariani A, Cimbanassi S. A population based study of hospitalised seriously injured in a region of Northern Italy. World J Emerg Surg 2013; 8: 32

3. Reilly PM, Schwab CW, Kauder DR, et al. The invisible trauma patient: emergency department discharges. J Trauma 2005; 58: 675-83. 
4. Markovchick VJ, Moore EE. Optimal trauma outcome: trauma system design and the trauma team. Emerg Med Clin N Am 2007; 25: 643-54.

5. Jacobs IA, Kelly K, Valenziano C, Chevinsky AH, Pawar J, Jones $C$. Cost savings associated with changes in routine laboratory tests ordered for victims of trauma. Am Surg 2000; 66: 579-84.

6. Salvagno GL, Sanchis-Gomar F, Picanza A, Lippi G. Red blood cell distribution width: a simple parameter with multiple clinical applications. Crit Rev Clin Lab Sci 2015; 52: 86-105.

7. Lippi G, Pavesi F, Bardi M, Pipitone S. Lack of harmonization of red blood cell distribution width (RDW). Evaluation of four hematological analyzers. Clin Biochem 2014; 47: 1100-3.

8. Danese E, Lippi G, Montagnana M. Red blood cell distribution width and cardiovascular diseases. J Thorac Dis 2015; 7: E402-11.

9. Lippi G, Buonocore R, Cervellin G. Value of red blood cell distribution width on Emergency Department admission in patients with venous thrombosis. Am J Cardiol 2016; 117: 670-5.

10. Ay S, Eryilmaz MA, Aksoy N, Okus A, Unlu Y, Sevinc B. Is early detection of colon cancer possible with red blood cell distribution width? Asian Pac J Cancer Prev 2015; 16: 753-6.

11. Kim CH, Park JT, Kim EJ, et al. An increase in red blood cell distribution width from baseline predicts mortality in patients with severe sepsis or septic shock. Crit Care 2013; 17: R282.

12. Patel KV, Ferrucci L, Ershler WB, Longo DL, Guralnik JM. Red blood cell distribution width and the risk of death in middle-aged and older adults. Arch Intern Med 2009; 169: 515-23.

13. Lippi G, Pavesi F, Bardi M, Pipitone S. Lack of harmonization of red blood cell distribution width (RDW). Evaluation of four hematological analyzers. Clin Biochem 2014; 47: 1100-3.

14. Derlet RW, McNamara RM, Kazzi AA, Richards JR. Emergency department crowding and loss of medical licensure: a new risk of patient care in hallways. West J Emerg Med 2014; 15: 137-41.

15. Sprivulis PC, Da Silva JA, Jacobs IG, Frazer AR, Jelinek GA The association between hospital overcrowding and mortality among patients admitted via Western Australian emergency departments. Med J Aust 2006; 184: 208-12.

16. Bamezai A, Melnick G, Nawathe A. The cost of an emergency department visit and its relationship to emergency department volume. Ann Emerg Med 2005; 45: 483-90.

17. Kim KH, Carey K, Burgess JF Jr. Emergency department visits: the cost of trauma centers. Health Care Manag Sci 2009; 12: 243-51.

18. Simonet D. Cost reduction strategies for emergency services: insurance role, practice changes and patients accountability. Health Care Anal 2009; 17: 1-19.

19. Lewandrowski K. How the clinical laboratory and the emergency department can work together to move patients through quickly. Clin Leadersh Manag Rev 2004; 18: 155-9.

20. Lippi G, Guidi GC. The power of negative thinking. Am J Emerg Med 2008; 26: 373-4.

21. Patel KV, Semba RD, Ferrucci L, et al. Red cell distribution width and mortality in older adults: a meta-analysis. J Gerontol A Biol Sci Med Sci 2010; 65: 258-65.
22. Majercik S, Fox J, Knight S, Horne BD. Red cell distribution width is predictive of mortality in trauma patients. J Trauma Acute Care Surg 2013; 74: 1021-6.

23. Garbharran U, Chinthapalli S, Hopper I, George M, Back $\mathrm{DL}$, Dockery F. Red cell distribution width is an independent predictor of mortality in hip fracture. Age Ageing 2013; 42: 258-61.

24. Zhang B, Zhao J. Red blood cell distribution width as a prognostic biomarker for mortality in traumatic brain injury. Int J Clin Exp Med 2015; 8: 19172-5.

25. Paulus EM, Weinberg JA, Magnotti LJ, et al. Admission red cell distribution width: a novel predictor of massive transfusion after injury. Am Surg 2014; 80: 685-9.

26. Yu HP, Chaudry IH, Choudhry MA, Hsing CH, Liu FC, Xia Z. Inflammatory response to traumatic injury: clinical and animal researches in inflammation. Mediators Inflamm 2015; 2015: 729637.

27. Rodríguez-Rodríguez A, Egea-Guerrero JJ, Murillo-Cabezas F, Carrillo-Vico A. Oxidative stress in traumatic brain injury. Curr Med Chem 2014; 21: 1201-11.

28. Wang M, Qiu W, Qiu F, Mo Y, Fan W. Tangent function transformation of the Abbreviated Injury Scale improves accuracy and simplifies scoring. Arch Med Sci 2015; 11: 130-6.

29. Kertmen H, Gürer B, Yilmaz ER, et al. Antioxidant and antiapoptotic effects of darbepoetin-alpha against traumatic brain injury in rats. Arch Med Sci 2015; 11: 1119-28.

30. Ou Z, Jiang Y, Wu BO, Duan YF, Sun ZD, Luo GH. Cystathionine-gamma-lyase inhibitor attenuates acute lung injury induced by acute pancreatitis in rats. Arch Med Sci 2014; 10: 825-9. 